NONCOMMUTATIVE GEOMETRY

AND QUANTUM GROUPS

BANACH CENTER PUBLICATIONS, VOLUME 61

INSTITUTE OF MATHEMATICS

POLISH ACADEMY OF SCIENCES

WARSZAWA 2003

\title{
QUANTUM 4-SPHERE: THE INFINITESIMAL APPROACH
}

\author{
F. BONECHI and M. TARLINI \\ INFN Sezione di Firenze and Dipartimento di Fisica, Università di Firenze, Firenze, Italy \\ E-mail: bonechi@fi.infn.it, tarlini@fi.infn.it \\ N. CICCOLI \\ Dipartimento di Matematica, Università di Perugia, Perugia, Italy \\ E-mail: ciccoli@dipmat.unipg.it
}

\begin{abstract}
We describe how the constructions of quantum homogeneous spaces using infinitesimal invariance and quantum coisotropic subgroups are related. As an example we recover the quantum 4-sphere of [2] through infinitesimal invariance with respect to $\mathcal{U}_{q}(S U(2))$.
\end{abstract}

1. Introduction. Since [9] it has been clear that quantum subgroups of a given quantum group are not enough to construct all examples of interesting embeddable quantum homogeneous spaces. This problem was first overcome by looking at infinitesimal invariants with respect to suitable $*$-subalgebras and right (or left) coideals of the universal enveloping algebra (thus weakening the condition of $*$-Hopf subalgebra). Although many families of quantum homogeneous spaces were constructed in this way ([6]) the geometrical meaning of this construction wasn't completely clear. Later in $[5,7]$ a new approach was considered in the context of quantum function algebras: looking at coinvariants with respect to two sided coideals and right (left) $(* \circ S)$-invariant ideals. In [7] this approach was motivated by an analysis of the underlying Poisson structure: any quotient Poisson structure is determined by a coisotropic subgroup of a given Poisson-Lie group. The two approaches turn out, in fact, to be equivalent; from this point of view $*$-subalgebras and right (or left) coideals of the universal enveloping algebra can be simply seen as tangent algebras of quantum coisotropic subgroups.

In this paper we review the theory of Poisson homogeneous spaces, we provide an interpretation of infinitesimal invariance and we describe the relation between the two quantum constructions. In the last section we explicitly describe the quantum 4-sphere of [2] in terms of infinitesimal invariance.

2000 Mathematics Subject Classification: 58B32, 53D17, $20 \mathrm{G} 42$.

The paper is in final form and no version of it will be published elsewhere. 
2. Poisson homogeneous spaces. In what follows $G$ will be a connected PoissonLie group with Poisson bivector $\omega$ and tangent Lie bialgebra $(\mathfrak{g}, \delta)$. Let us recall that a Lie subgroup $H$ of $G$ is called a Poisson-Lie subgroup if the identity map $H \hookrightarrow G$ is a Poisson map.

Let now $\left(M, \omega_{M}\right)$ be a Poisson manifold and $\phi: G \times M \rightarrow M$ an action of $G$ on $M$. We will use the following notation:

$$
\phi_{x}(g)=\phi(g, x)=\phi_{g}(x)=g \cdot x \quad \forall x \in M, \forall g \in G .
$$

Definition 2.1. A Poisson manifold $\left(M, \omega_{M}\right)$ is a $\left(G, \omega_{G}\right)$-Poisson homogeneous space if there is a smooth homogeneous action $\phi: G \times M \rightarrow M$ which is a Poisson map with respect to the product Poisson structure. In this case we will also say that $\omega_{M}$ is $\left(G, \omega_{G}\right)$-covariant.

In terms of Poisson bivectors, covariance is equivalent to:

$$
\omega_{M}(g x)=\left(\phi_{x}\right)_{*, g} \omega_{G}(g)+\left(\phi_{g}\right)_{*, x} \omega_{M}(x) \quad \forall x \in M, g \in G .
$$

If $(G, 0)$ is the trivial Poisson-Lie group this simply means that the Poisson bivector $\omega_{M}$ is $G$-invariant, i.e.

$$
\phi_{*, g} \omega_{M}=\omega_{M} \quad \forall g \in G .
$$

It is well known that $G$-homogeneous spaces are in bijective correspondence with conjugacy classes of closed subgroups of $G$. This correspondence breaks down at the Poisson level due to non-invariance under conjugation of the Poisson-subgroup property. However some Poisson homogeneous spaces still correspond to subgroups having a natural behaviour with respect to the Poisson structure. A coisotropic subgroup is defined as a closed subgroup of a Poisson-Lie group which is also a coisotropic submanifold (i.e. a connected submanifold whose defining ideal is a Poisson subalgebra in $C^{\infty}(G)$, more details in [15]).

Proposition 2.2 ([10]). Let $G$ be a Poisson-Lie group and $H$ be a connected subgroup with Lie algebra $\mathfrak{h} \subseteq \mathfrak{g}$. The following are equivalent:

i) $\delta(\mathfrak{h}) \subset \mathfrak{g} \wedge \mathfrak{h}$;

ii) $\mathfrak{h}^{\perp}$ is a Lie subalgebra of $\mathfrak{g}^{*}$;

iii) $H$ is a coisotropic subgroup of $G$.

The role of coisotropic subgroups in the theory of Poisson homogeneous spaces is well explained by the following proposition.

Proposition 2.3. Let $M$ be a G-Poisson homogeneous space with action $\sigma$. The following are equivalent:

1. there exists $x_{0} \in M$ such that the stabilizer $G_{x_{0}}$ is coisotropic in $G$;

2. there exists $x_{0}$ in $M$ such that $\sigma_{x_{0}}: G \rightarrow M$ is a Poisson map;

3. there exists $x_{0}$ in $M$ at which $\omega_{M}\left(x_{0}\right)=0$.

Definition 2.4. We will say that $\left(M, \omega_{M}\right)$ is a coisotropic G-homogeneous space if it is a $G$-Poisson homogeneous space, there exists a coisotropic closed subgroup $H$ of $G$ 
such that $M \simeq G / H$ as a manifold and the natural projection $\pi: G \rightarrow G / H$ satisfies

$$
\omega_{M}(g H)=\pi_{*, g} \omega_{G}(g) \quad \forall g \in G .
$$

Remark, then, that if one considers the universal enveloping algebra $\mathcal{U}(\mathfrak{g})$ with its natural co-Poisson Hopf structure $([6])$ its natural subalgebra $\mathcal{U}(\mathfrak{h})$ is not a co-Poisson Hopf subalgebra but just a Hopf subalgebra such that $\delta(\mathcal{U}(\mathfrak{h})) \subseteq \mathcal{U}(\mathfrak{h}) \wedge \mathcal{U}(\mathfrak{g})$, i.e. it is a Lie coideal.

An interesting family of coisotropic homogeneous spaces is given by group-like symmetric spaces. Let, in fact, $G$ be a Poisson-Lie group and denote with $\bar{G}$ the same group with opposite Poisson structure. It can be proven (see [15]) that the diagonal inclusion of $G$ in $G \times \bar{G}$ gives $G$ the structure of a coisotropic subgroup of $G \times \bar{G}$ and thus $G \times \bar{G} / G \simeq G$ is always a coisotropic homogeneous space.

Let $G^{*}$ be the unique connected simply connected dual group of the Poisson-Lie group $(G, \omega)$. Then, from proposition 2.2 , to every connected coisotropic subgroup $H$ of $G$ we can associate a unique coisotropic connected subgroup $H^{\perp}$ of $G^{*}$ that we will call complementary dual of $H$.

Remark that every time we have a coisotropic $G$-homogeneous space we can construct a coisotropic $G^{*}$-homogeneous space $G^{*} / H^{\perp}$. We will call it the complementary dual homogeneous space of $G / H$. Quantization of such spaces can be constructed relying on the so called quantum duality principle. Details will be given in a forthcoming paper by one of the authors [8].

It is clear that the tangent space $T_{x}\left(G^{*} / H^{\perp}\right)$ can be identified with the space of tangent vector of $G^{*}$ which are zero in the direction of $H^{\perp}$ and so with $\mathfrak{h} \simeq \mathfrak{g}^{*} / \mathfrak{h}^{\perp}$.

3. Quantum coisotropic subgroups. The theory of Poisson-homogeneous spaces, as outlined in the previous section, can be seen as a guideline in building a theory of quantum homogeneous spaces. If quantization is seen as a functor from Poisson-Lie groups to non-commutative topological Hopf-algebras then every quantum algebra map at the semiclassical level should define a Poisson map. Thus embeddable quantum homogeneous spaces (see Definition 3.1 later on), which always possess one character (the restriction of the counit), quantize those Poisson homogeneous spaces which always possess a 0 -dimensional singleton leaf, which in view of proposition 2.3 are exactly coisotropic homogeneous spaces. Unfortunately the quantization procedure can be proved to be a functor only under quite restrictive settings.

Definition $3.1([9])$. Let $\mathcal{F}_{q}(G)$ be a Hopf-*-algebra. A right embeddable quantum homogeneous space of $\mathcal{F}_{q}(G)$ is a $*$-subalgebra $B$ of $\mathcal{F}_{q}(G)$ which is also a right coideal.

The general theory that follows is valid for any Hopf-*-algebra. However the class of algebras we're interested in is the more restrictive one of quantum groups, i.e. Hopf-*algebras $\mathcal{F}_{q}(G)$ deforming commutative algebras of functions on algebraic groups. In this case the group is naturally endowed with a multiplicative Poisson structure to which we will refer as the semiclassical limit. From now on $\mathcal{F}_{q}(G)$ will be tacitly assumed to be of this kind. 
Definition $3.2([3],[7])$. For a given quantum group $\mathcal{F}_{q}(G)$, a coisotropic quantum right (left) subgroup is every coalgebra $C$ such that:

1. $C$ is a right (left) $\mathcal{F}_{q}(G)$-module;

2. there exists a surjective linear map $\pi: \mathcal{F}_{q}(G) \rightarrow C$ which is a morphism of $\mathcal{F}_{q}(G)$ modules (where $\mathcal{F}_{q}(G)$ is considered as a module over itself via multiplication) and of coalgebras.

If $\mathcal{F}_{q}(G)$ has a real structure * (i.e. an involutive algebra antihomomorphism and coalgebra homomorphism) we say that a coisotropic quantum right (left) subgroup $C$ is real if there exists an involution $\tau_{C}: C \rightarrow C$ such that

$$
\tau_{C} \circ \pi=\pi \circ(* \circ S) \text {. }
$$

Remark that every quantum coisotropic subgroup has a distinguished group-like element $\pi(1)$. Furthermore the involution $\tau_{C}$ satisfies

$$
\begin{gathered}
\Delta\left(\tau_{C}(c)\right)=\sigma_{1,2}\left(\tau_{C} \otimes \tau_{C}\right) \Delta(c), \quad \forall c \in C, \\
\varepsilon\left(\tau_{C}(c)\right)=\overline{\varepsilon(c)} .
\end{gathered}
$$

We also remark here that not every coisotropic quantum subgroup can be given a compatible Hopf structure as shown in [4].

Proposition 3.3. There exists a bijective correspondence between coisotropic quantum right (left) subgroups and two-sided coideals which are also right (left) ideals in $\mathcal{F}_{q}(G)$, given by associating to $C$ the kernel of the projection $\pi$. In this correspondence real quantum coisotropic subgroups will correspond to $(* \circ S)$-invariant ideals.

The kernel of $\pi$ is often called the defining ideal of $C$. It has to be noted that the idea of quantum coisotropic subgroup was known to Hopf algebraists before quantum groups came into play, simply as right ideals and two-sided coideals. We prefer, however, to keep this name to emphasize the analogy with the Poisson case. In examples, like the one to be used later on, it would have been very difficult to guess the right two-sided coideal without any guidance from the Poisson theory.

Let us remark that although it is quite easy to verify that the semiclassical limit of a quantum coisotropic subgroup is a coisotropic subgroup, it is by no means clear that every coisotropic subgroup admits quantization. Even if no counterexample is known such a definition does not allow, at present, to establish a bijective correspondence between classical and quantum coisotropic subgroups.

Proposition 3.4. Let $C$ be a right coisotropic quantum subgroup in $\mathcal{F}_{q}(G)$, with defining ideal $\mathcal{I}$ and projection map $\pi: \mathcal{F}_{q}(G) \rightarrow C$. The set of coinvariant functions:

$$
B_{C}=B_{\mathcal{I}}=\left\{b \in \mathcal{F}_{q}(G) \mid(\mathrm{id} \otimes \pi) \circ \Delta b=b \otimes \pi(1)\right\}
$$

is an embeddable quantum homogeneous space.

Let now $B$ be an embeddable quantum homogeneous space and define

$$
\mathcal{I}_{B}=\left\{\sum_{j}\left[b_{j}-\varepsilon\left(b_{j}\right) 1\right] u_{j} \mid b_{j} \in B, u_{j} \in \mathcal{F}_{q}(G)\right\},
$$

i.e. the right ideal generated by all elements $\left(b_{j}-\varepsilon\left(b_{j}\right)\right)$ such that $b_{j} \in B$. 
Proposition 3.5. $\mathcal{I}_{B}$ is a two-sided coideal and a right ideal.

If $B$ is a $\mathcal{F}_{q}(G)$-quantum embeddable homogeneous space we will call the quotient coalgebra $\mathcal{F}_{q}(G) / \mathcal{I}_{B}$ together with the obvious projection map, the coisotropic quantum subgroup of $\mathcal{F}_{q}(G)$ associated to $B$.

Definition 3.6. A quantum coisotropic subgroup $C$ of $\mathcal{F}_{q}(G)$ is said to be stable if its defining ideal $\mathcal{I}$ satisfies $\mathcal{I}=\mathcal{I}_{B_{\mathcal{I}}}$. Similarly an embeddable quantum homogeneous space $B$ is said to be stable if $B=B_{\mathcal{I}_{B}}$.

In $[12,13]$ one can find details about the algebraic conditions under which stability is verified. It depends on $\mathcal{F}_{q}(G)$ being faithfully flat over $B$ or on $\mathcal{F}_{q}(G)$ being faithfully coflat over $C$. This establishes a bijective correspondence between suitable subclasses of quantum coisotropic subgroups and embeddable quantum homogeneous space as explained, for example, in [12].

Let's now move to the enveloping algebra level. First of all we will need Hopf- $*$-algebra pairings; for a detailed exposition of their properties we refer to [14].

Definition 3.7. Let $\mathcal{F}_{q}(G)$ and $\mathcal{U}_{q}(G)$ be two Hopf-*-algebras. A pairing is a bilinear map

$$
\langle., .\rangle: A \times U \rightarrow \mathbb{C}
$$

such that:

1. $\left\langle\Delta a, u_{1} \otimes u_{2}\right\rangle=\left\langle a, u_{1} u_{2}\right\rangle, \forall a \in A, u_{1}, u_{2} \in U$;

2. $\left\langle a_{1} \otimes a_{2}, \Delta u\right\rangle=\left\langle a_{1} a_{2}, u\right\rangle$

3. $\langle a, S(u)\rangle=\langle S(a), u\rangle$;

4. $\left\langle a^{*}, u\right\rangle=\overline{\left\langle a, S(u)^{*}\right\rangle}$

Let $\mathcal{F}_{q}(G)$ and $\mathcal{U}_{q}(G)$ be Hopf-*-algebras in non-degenerate duality in what follows. Let us also consider this more general situation.

DeFinition 3.8. Let $C$ be a coalgebra and a right $\mathcal{F}_{q}(G)$-module. Let $V$ be an unital algebra and a right $\mathcal{U}_{q}(G)$-comodule. A semipairing between $C$ and $V$ is a linear mapping

$$
\langle\langle., .\rangle\rangle: C \otimes V \rightarrow \mathbb{C}
$$

such that:

i) $\left\langle\left\langle\Delta c, v_{1} \otimes v_{2}\right\rangle\right\rangle=\left\langle\left\langle c, v_{1} v_{2}\right\rangle\right\rangle$, for all $c \in C, v_{1}, v_{2} \in V$;

ii) $\langle\langle c, 1\rangle\rangle=\varepsilon(c)$, for all $c \in C$.

It is called a pairing if it furthermore satisfies:

iii) $\langle\langle c \cdot f, v\rangle\rangle=\sum_{(v)}\left\langle\left\langle c, v_{(1)}\right\rangle\right\rangle\left\langle f, v_{(2)}\right\rangle$ where $f \in \mathcal{F}_{q}(G)$ and $\langle$,$\rangle is the non-degenerate$ pairing of $\mathcal{F}_{q}(G)$ and $\mathcal{U}_{q}(G)$.

Lastly, if $C$ is real with involution $\tau$ we require also

iv) There exists a $*$-algebra structure on $V$ such that $\overline{\langle\langle\tau(c), f\rangle\rangle}=\left\langle\left\langle c, f^{*}\right\rangle\right\rangle$.

Recall that there is a natural left action of $\mathcal{U}_{q}(G)$ on $\mathcal{F}_{q}(G)$ given by:

$$
u \cdot a=(\mathrm{id} \otimes u) \circ \Delta(a)=\sum_{(a)}\left\langle a_{(2)}, u\right\rangle a_{(1)} .
$$


An element $a \in \mathcal{F}_{q}(G)$ such that $u \cdot a=\varepsilon(u) a$ is said to be infinitesimally invariant with respect to $u \in \mathcal{U}_{q}(G)$.

Proposition 3.9. Let $J$ be a $(* \circ S)$-invariant two-sided coideal and left ideal in $\mathcal{U}_{q}(G)$. Then $B_{J}=\left\{a \in \mathcal{F}_{q}(G) \mid J \cdot a=0\right\}$ is a*-subalgebra and left coideal in $\mathcal{F}_{q}(G)$. Furthermore

$$
B_{J}=\left\{a \in \mathcal{F}_{q}(G) \mid\langle a, j\rangle=0 \forall j \in J\right\} .
$$

Let $B$ be $a *$-subalgebra and left coideal in $\mathcal{F}_{q}(G)$. Then the set $J_{B}=\left\{u \in \mathcal{U}_{q}(G) \mid u \cdot b=\right.$ $0 \forall b \in B\}$ is a left ideal and a $\tau$-invariant two-sided coideal in $\mathcal{U}_{q}(G)$. Furthermore

$$
J_{B}=\left\{u \in \mathcal{U}_{q}(G) \mid\langle b, u\rangle=0 \forall b \in B\right\} .
$$

Coideal subalgebras in quantum enveloping algebras, which were used to find embeddable quantum homogeneous spaces not obtained by quantum subgroups, see for instance [9], can then be interpreted as tangent algebras of quantum coisotropic subgroups as defined in what follows.

Let now $(C, \pi)$ be a right coisotropic quantum subgroup of $\mathcal{F}_{q}(G)$.

DeFinition 3.10. If there exists a $*$-algebra $V$ which is also a right $\mathcal{U}_{q}(G)$-comodule together with a non degenerate pairing between $V$ and $C$ (in the sense of 3.8), then $V$ is called the tangent algebra of $(C, \pi)$.

If, furthermore, there exists $\psi: V \rightarrow \mathcal{U}_{q}(G)$ such that:

1. $\psi$ is a $*$-algebra morphism;

2. $\psi$ is a $\mathcal{U}_{q}(G)$-comodule morphism;

3. $\psi$ is dual to the projection $\pi$, i.e.

$$
\langle f, \psi(v)\rangle=\langle\langle\pi(f), v\rangle\rangle \quad \forall v \in V \quad \forall f \in \mathcal{F}_{q}(G)
$$

then $V$ is called an embeddable tangent algebra of $(C, \pi)$.

Let us remark that non-degeneracy of the pairing implies that $\psi$ is injective. Furthermore there is a 1:1 correspondence between such $\psi$ 's and the set of $*$-algebra homomorphisms $\varepsilon: V \rightarrow \mathbb{C}$. This clarifies in which sense two embeddable tangent algebras of the same coisotropic subgroup can differ: they are different embeddings of isomorphic $*$-algebras and right $\mathcal{U}_{q}(G)$-comodules. Let us consider the semiclassical limit of a *-subalgebra right (or left) coideal in $\mathcal{U}_{q}(G)$. This means taking into account the natural co-Poisson Hopf algebra structure on $\mathcal{U}(\mathfrak{g})$ given by the first order in $\Delta$. With respect to this structure it is evident that an embedded tangent algebra goes in the enveloping algebra of the tangent Lie algebra of a coisotropic subgroup. This gives an interpretation to the otherwise quite mysterious one-sided coideal property.

Let $(C, \pi)$ be a coisotropic quantum subgroup of $\mathcal{F}_{q}(G)$ with an embeddable tangent algebra $(V, \psi)$. Define $J_{V}$ to be the right ideal in $\mathcal{U}_{q}(G)$ generated by the set $\{\psi(v)-\varepsilon(\psi(v)) 1 \mid v \in V\}$ (i.e. the kernel of the counit restricted to $\psi(V))$.

Proposition 3.11. $J_{V}$ is a two-sided coideal in $\mathcal{U}_{q}(G)$.

Proposition 3.12. The space of $\mathcal{F}_{q}(G)$-coinvariants with respect to $C$ coincides with the space of infinitesimal invariants with respect to $J_{V}$, i.e. $B_{C}=B_{J_{V}}$. 
The proof of Proposition 3.12 is the same of Proposition 1.12 of [9]. Just remark that the rather obscure existence condition for a map $\psi$ stated there can be interpreted, in the present setting, as the embeddability for the dual space.

Thus we've seen that any embeddable quantum homogeneous space obtained by a coisotropic quantum subgroup can also be constructed through infinitesimal invariance, provided the coisotropic subgroup has an embeddable tangent algebra.

There are analogous results concerning left coisotropic subgroups and their homogeneous spaces. It can be easily seen that they imply a similar construction for double cosets and we will use it in the following section.

4. The quantum 4-sphere. Many new non-commutative 4-spheres were constructed in different contexts. Some of them can be related to quantum groups, as explained also in [1]. In this section we will show how the quantum 4-sphere, constructed from a suitable quantum coisotropic subgroup of $\mathcal{F}_{q}(U(4))$ in [2] (to which we refer for details), can be described using infinitesimal invariance. The setting will not be exactly the same as in the previous section. The 4 -sphere is, in fact a double coset, obtained by a $\mathcal{F}_{q}(S U(2))$ coaction on the seven sphere $\mathcal{F}_{q}\left(\mathbb{S}^{7}\right)=\mathcal{F}_{q}(U(3) \backslash U(4))$. At an algebraic level this means that coinvariants and infinitesimal invariants should be considered only inside the *subalgebra $\mathcal{F}_{q}\left(\mathbb{S}^{7}\right)$, rather than in the whole $\mathcal{F}_{q}(U(4))$. This does not affect significantly the relation between the two constructions.

The Hopf algebra $\mathcal{F}_{q}(U(4))$ is generated by $\left\{t_{i j}\right\}_{i j=1}^{4}, D_{q}^{-1}$ and the following relations (see [11]):

$$
\begin{array}{rlr}
t_{i k} t_{j k}=q t_{j k} t_{i k}, & t_{k i} t_{k j}=q t_{k j} t_{k i}, & i<j, \\
t_{i \ell} t_{j k} & =t_{j k} t_{i \ell}, \quad i<j, k<\ell, \\
t_{i k} t_{j \ell}-t_{j \ell} t_{i k} & =\left(q-q^{-1}\right) t_{j k} t_{i \ell}, & i<j, k<\ell, \\
D_{q} D_{q}^{-1} & =D_{q}^{-1} D_{q}=1,
\end{array}
$$

where $D_{q}=\sum_{\sigma \in P_{4}}(-q)^{\ell(\sigma)} t_{\sigma(1) 1} \ldots t_{\sigma(4) 4}$ with $P_{4}$ being the group of 4-permutations, is central. Motivated by the analysis of coisotropic subgroups of standard $U(4)$ (see [2]) let us define $\mathcal{R}=R \mathcal{F}_{q}(U(4))$, where

$$
\begin{gathered}
R=\operatorname{Span}\left\{t_{13}, t_{31}, t_{14}, t_{41}, t_{24}, t_{42}, t_{23}, t_{32}, t_{11}-t_{44}, t_{12}+t_{43},\right. \\
\left.t_{21}+t_{34}, t_{22}-t_{33}, t_{11} t_{22}-q t_{12} t_{21}-1\right\} .
\end{gathered}
$$

It is easy to verify that $\mathcal{R}$ is a $\tau$-invariant, right ideal, two-sided coideal. Let $r: \mathcal{F}_{q}(U(4))$ $\rightarrow \mathcal{F}_{q}(U(4)) / \mathcal{R}$ be the projection map. We have the following result:

Proposition 4.1. As a $\tau$-coalgebra, $\mathcal{F}_{q}(U(4)) / \mathcal{R}$ is isomorphic to $\mathcal{F}_{q}(S U(2))$.

As explained in $[2], \mathcal{F}_{q}(U(4)) / \mathcal{R}$ quantizes a subgroup conjugate to the diagonal $S U(2)$.

REMARK 1. The projection map $r: \mathcal{F}_{q}(U(4)) \rightarrow \mathcal{F}_{q}(S U(2))$ is not a Hopf algebra map as can be, for instance, explicitly verified on $r\left(t_{11} t_{43}\right) \neq r\left(t_{11}\right) r\left(t_{43}\right)$. Anyway $r$ defines a right $\mathcal{F}_{q}(U(4))$-module structure on the quotient.

The algebra $\mathcal{F}_{q}\left(\mathbb{S}^{7}\right) \hookrightarrow \mathcal{F}_{q}(U(4))$ is generated by $z_{i}=t_{4 i}, i=1, \ldots, 4$, with the following relations [16]:

$$
z_{i} z_{j}=q z_{j} z_{i}(i<j), \quad z_{j}^{*} z_{i}=q z_{i} z_{j}^{*} \quad(i \neq j)
$$




$$
z_{k}^{*} z_{k}=z_{k} z_{k}^{*}+\left(1-q^{2}\right) \sum_{j<k} z_{j} z_{j}^{*}, \quad \sum_{k=1}^{4} z_{k} z_{k}^{*}=1 .
$$

This algebra can be identified with the algebra of coinvariant functions with respect to the standard quantum subgroup $\mathcal{F}_{q}(U(3))$ of $\mathcal{F}_{q}(U(4))$ and therefore carries a natural $\mathcal{F}_{q}(U(4))$-coaction by restriction of the coproduct: $\Delta: \mathcal{F}_{q}\left(\mathbb{S}^{7}\right) \rightarrow \mathcal{F}_{q}\left(\mathbb{S}^{7}\right) \otimes \mathcal{F}_{q}(U(4))$. By composing with the coalgebra projection $r, \Delta_{r}=(\mathrm{id} \otimes r) \Delta: \mathcal{F}_{q}\left(\mathbb{S}^{7}\right) \rightarrow \mathcal{F}_{q}\left(\mathbb{S}^{7}\right) \otimes$ $\mathcal{F}_{q}(S U(2))$ defines an $\mathcal{F}_{q}(S U(2))$ coaction on $\mathcal{F}_{q}\left(\mathbb{S}^{7}\right)$. The space of functions on the quantum 4 -sphere $\Sigma_{q}^{4}=\mathcal{F}_{q}\left(\mathbb{S}^{4}\right)$ is the space of coinvariants with respect to this coaction, i.e. $\Sigma_{q}^{4}=\left\{a \in \mathcal{F}_{q}\left(\mathbb{S}^{7}\right) \mid \Delta_{r}(a)=a \otimes r(1)\right\}$.

Proposition 4.2. The algebra $\Sigma_{q}^{4}$ is generated by $\left\{a, a^{*}, b, b^{*}, R\right\}$, where $a=z_{1} z_{4}^{*}-$ $z_{2} z_{3}^{*}, \quad b=z_{1} z_{3}+q^{-1} z_{2} z_{4}, R=z_{1} z_{1}^{*}+z_{2} z_{2}^{*}$. They satisfy the following relations

$$
\begin{gathered}
R a=q^{-2} a R, \quad R b=q^{2} b R, \quad a b=q^{3} b a, \quad a b^{*}=q^{-1} b^{*} a, \\
a a^{*}+q^{2} b b^{*}=R\left(1-q^{2} R\right), \\
a a^{*}=q^{2} a^{*} a+\left(1-q^{2}\right) R^{2}, \quad b^{*} b=q^{4} b b^{*}+\left(1-q^{2}\right) R .
\end{gathered}
$$

Let's now move to the tangent algebra of the coisotropic quantum subgroup $\mathcal{F}_{q}(S U(2))$. Let us consider now the simply connected form of the universal enveloping algebra $\mathcal{U}_{q}(u(4))$; its algebra generators are $K_{i}^{1 / 2}, K_{i}^{-1 / 2}, E_{i}, F_{i}, i=1, \ldots, 4$. Let us recall the relations

$$
\begin{gathered}
K_{i}^{1 / 2} E_{j} K_{i}^{-1 / 2}=q^{a_{i j} / 2} E_{j}, \quad K_{i}^{1 / 2} F_{j} K_{i}^{-1 / 2}=q^{-a_{i j} / 2} F_{j}, \\
{\left[E_{i}, F_{j}\right]=\delta_{i j} \frac{K_{i}-K_{i}^{-1}}{q-q^{-1}}, K_{i}^{1 / 2} K_{j}^{1 / 2}=K_{j}^{1 / 2} K_{i}^{1 / 2},}
\end{gathered}
$$

which will be used in what follows ([6] for the full list of relations). Its Hopf-*-algebra structure is completed by:

$$
\begin{aligned}
& \Delta\left(E_{i}\right)=E_{i} \otimes K_{i}+1 \otimes K_{i}, \Delta\left(F_{i}\right)=F_{i} \otimes 1+K_{i}^{-1} \otimes F_{i} \\
& \Delta\left(K_{i}\right)=K_{i} \otimes K_{i}, \\
& S\left(E_{i}\right)=-E_{i} K_{i}, \quad S\left(K_{i}\right)=K_{i}^{-1}, \quad S\left(F_{i}\right)=-K_{i} F_{i}, \\
& E_{i}^{*}=K_{i} F_{i}, \quad K_{i}^{*}=K_{i}, \quad F_{i}^{*}=E_{i} K_{i}^{-1} .
\end{aligned}
$$

The fundamental unitary four dimensional representation $T$ is defined as:

$$
\begin{gathered}
T\left(K_{i}\right)=q^{-1} e_{i i}+q e_{i+1, i+1}+\sum_{j \neq i, i+1} e_{j j}, \\
T\left(E_{i} K_{i}^{-1 / 2}\right)=e_{i+1, i}, \quad T\left(K_{i}^{1 / 2} F_{i}\right)=e_{i, i+1} .
\end{gathered}
$$

Here $e_{i, j}$ are the usual matrices with 1 on the $i, j$-entry and 0 elsewhere.

The non-degenerate Hopf algebra duality paring between $\mathcal{U}_{q}(u(4))$ with $\mathcal{F}_{q}(U(4))$ can be explicitly given by:

$$
\left\langle X, t_{i j}\right\rangle=T(X)_{i j}, \quad X \in \mathcal{U}_{q}(u(4)) .
$$

Let's now define the tangent algebra of the coisotropic quantum subgroup $\mathcal{F}_{q}(S U(2))$ as

$$
\mathcal{U}_{q}(s u(2))=\left\{X \in \mathcal{U}_{q}(u(4)) \mid\langle X, t\rangle=0 \quad \forall t \in \operatorname{ker}(r)\right\}
$$


Consider the $*$-subalgebra $V$ of $\mathcal{U}_{q}(u(4))$ generated by:

$$
K=K_{1} K_{3}^{-1}, \quad E=E_{1} K^{-1 / 2}-\left(K_{1} K_{3}\right)^{1 / 2} F_{3}, \quad F=K_{1} K_{3}^{1 / 2} F_{1}-E_{3} K^{1 / 2} .
$$

It is an easy exercise to compute the following coproducts:

$$
\begin{gathered}
\Delta(E)=E \otimes\left(K_{1} K_{3}\right)^{1 / 2}+K^{-1 / 2} \otimes E+\left(K^{-1 / 2}-K^{1 / 2}\right) \otimes\left(K_{1} K_{3}\right)^{1 / 2} F_{3} \\
\Delta(F)=F \otimes\left(K_{1} K_{3}\right)^{1 / 2}+K^{-1 / 2} \otimes F+\left(K^{-1 / 2}-K^{1 / 2}\right) \otimes E_{3} K^{1 / 2} \\
\Delta(K)=K \otimes K .
\end{gathered}
$$

Then $V$ acts on $\mathcal{F}_{q}(U(4))$ as follows:

$$
\begin{aligned}
E \cdot t_{i j} & =\delta_{j 1} t_{i 2}-\delta_{j 4} t_{i 3}, \\
F \cdot t_{i j} & =\delta_{j 2} t_{i 1}-\delta_{j 3} t_{i 4}, \\
K^{ \pm 1} \cdot t_{i j} & =q^{ \pm \sigma(j)} t_{i j},
\end{aligned}
$$

where $\sigma(j)=-1$ if $j=1,4$ and $\sigma(j)=1$, if $j=2,3$.

Proposition 4.3. The algebra $V$ is the tangent algebra of $\mathcal{F}_{q}(S U(2))$ isomorphic to $\mathcal{F}_{q}(U(4)) / \mathcal{R}$. As an algebra it is isomorphic to the standard $\mathcal{U}_{q}(s u(2))$.

A direct computation shows, in fact, that $K, E, F$ kill all elements in $\operatorname{ker}(r)$, implying $V \subseteq \mathcal{U}_{q}(s u(2))$ and that they satisfy the usual $\mathcal{U}_{q}(s u(2))$ algebra relations:

$$
\begin{gathered}
K E=q^{2} E K, \quad K F=q^{-2} F K, \\
{[E, F]=\frac{K-K^{-1}}{q-q^{-1}} .}
\end{gathered}
$$

To prove that $V$ coincides with the whole tangent algebra $\mathcal{U}_{q}(s u(2))$ one has to proceed analogously to the appendix of [2] and consider the regular decomposition of $\mathcal{F}_{q}(U(4))$ under the left action of $\mathcal{U}_{q}(S U(2))$.

\section{References}

[1] P. Aschieri and F. Bonechi, On the noncommutative geometry of twisted spheres, Lett. Math. Phys. 59 (2002), 133-156.

[2] F. Bonechi, N. Ciccoli and M. Tarlini, Non commutative instantons on the 4-sphere from quantum groups, Comm. Math. Phys. 226 (2002), 419-432.

[3] F. Bonechi, N. Ciccoli, R. Giachetti, E. Sorace and M. Tarlini, Unitarity of induced representations from coisotropic quantum subgroups, Lett. Math. Phys. 49 (1999), 17-31.

[4] F. Bonechi, N. Ciccoli, R. Giachetti, E. Sorace and M. Tarlini, The coisotropic subgroup structure of quantum $S L(2, \mathbb{R})$, J. Geom. Phys. 37 (2001), 190-200.

[5] T. Brzeziński, Quantum homogeneous spaces as quantum quotient spaces, J. Math. Phys. 37 (1996), 2388-2399.

[6] V. Chari and A. Pressley, A Guide to Quantum Groups, Cambridge Univ. Press, Cambridge, 1994.

[7] N. Ciccoli, Quantization of coisotropic subgroups, Lett. Math. Phys. 42 (1997), 123-138.

[8] N. Ciccoli and F. Gavarini, The quantum duality principle for embeddable quantum homogeneous spaces, preprint, 2002. 
[9] M. S. Dijkhuizen and T. H. Koornwinder, Quantum homogeneous spaces, duality and the quantum 2-spheres, Geom. Ded. 52 (1994), 291-315.

[10] J.-H. Lu and A. Weinstein, Poisson-Lie groups, dressing transformations and Bruhat decomposition, J. Diff. Geom. 31 (1990), 501-526.

[11] A. Klimyk and K. Schmüdgen, Quantum Groups and Their Representations, Springer, Berlin, 1997.

[12] A. Masuoka, Quotient theory for Hopf algebras, in: Advances in Hopf Algebras, Lecture Notes in Pure Appl. Math. 158, Dekker, New York, 1994.

[13] E. F. Müller and H.-J. Schneider, Quantum homogeneous spaces with faithfully flat module structures, Israel J. Math. 31 (1999), 501-526.

[14] A. Van Daele, Dual pairs of Hopf-*-algebras, Bull. London Math. Soc. 205 (1993), 209-230.

[15] I. Vaisman, Geometry of Poisson Manifolds, Progress in Math. 118, Birkhäuser, 1994.

[16] L. L. Vaksman and Ya. Soibelman, Algebra of functions on the quantum group $S U(N+1)$ and odd dimensional quantum spheres, Leningrad Math. J. 2 (1991), 1023-1042. 